\title{
Polymorphisms in CXCR3 ligands predict early CXCL9 recovery and severe chronic GVHD
}

\author{
Hao Dai ${ }^{1}$, Sivaramakrishna P. Rachakonda ${ }^{1}$, Olaf Penack², Igor W. Blau², Olga Blau², Aleksandar Radujkovic ${ }^{3}$, \\ Carsten Müller-Tidow ${ }^{3}$, Peter Dreger $\mathbb{1 0}^{3}$, Rajiv Kumar ${ }^{1}$ and Thomas Luft $\mathbb{B}^{3}$
}

\begin{abstract}
Chronic graft-versus-host disease (cGVHD) is a major cause of mortality and morbidity after allogeneic stem cell transplantation (alloSCT). The individual risk of severe CGVHD remains difficult to predict and may involve CXCR3 ligands. This study investigated the role of single-nucleotide polymorphisms (SNPS) of CXCL4, CXCL9, CXCL10, and CXCL11, and their day +28 serum levels, in CGVHD pathogenesis. Eighteen CXCR3 and CXCL4, CXCL9-11 SNPs as well as peri-transplant CXCL9-11 serum levels were analyzed in 688 patients without (training cohort; $n=287$ ) or with statin-based endothelial protection cohort $(n=401)$. Clinical outcomes were correlated to serum levels and SNP status. Significant polymorphisms were further analyzed by luciferase reporter assays. Findings were validated in an independent cohort $(n=202)$. A combined genetic risk comprising four CXCR3 ligand SNPs was significantly associated with increased risk of severe cGVHD in both training cohort (hazard ratio (HR) 2.48, 95\% confidence interval (Cl) $1.33-4.64, P=0.004$ ) and validation cohort (HR 2.95, 95\% Cl 1.56-5.58, $P=0.001$ ). In reporter assays, significantly reduced suppressive effects of calcineurin inhibitors in constructs with variant alleles of rs884304 $(P<0.001)$ and rs884004 $(P<0.001)$ were observed. CXCL9 serum levels at day +28 after alloSCT correlated with both genetic risk and risk of severe CGVHD (HR 1.38, 95\% Cl 1.10-1.73, $P=$ 0.006). This study identifies patients with high genetic risk to develop severe cGVHD.
\end{abstract}

\section{Introduction}

Chronic graft-versus-host disease (cGVHD) is a leading cause of morbidity and mortality after allogeneic stem cell transplantation (alloSCT) ${ }^{1-3}$. cGVHD encompasses dysregulated immune responses, chronic inflammation, and fibrosis, with various manifestations resembling autoimmune diseases ${ }^{4}$. Steroid-based immunosuppressive therapy achieves meaningful responses in only $50 \%$ of the patients ${ }^{5,6}$. cGVHD can be graded as severe or non-severe cGVHD according to the National Institutes of Health (NIH) consensus criteria ${ }^{7}$. Non-severe cGVHD is associated with better overall survival because of an increased graft-versus-leukemia (GVL) effect, while severe cGVHD

\footnotetext{
Correspondence: Thomas Luft (Thomas.luft@med.uni-heidelberg.de)

${ }^{1}$ Department of Epidemiology, German Cancer Research Centre (DKFZ), Heidelberg, Germany

${ }^{2}$ Division of Hematology, Oncology and Tumorimmunology, Charité University Medicine Berlin, Berlin, Germany
}

Full list of author information is available at the end of the article causes significant morbidity and adverse impact on survival in long-term survivors ${ }^{8}$. Therefore, better prediction algorithms of severe cGVHD might improve risk-benefit assessments for patients before undergoing alloSCT.

Accumulating evidence suggests that type $1 \mathrm{~T}$ helper (Th1) cells play a critical role in the pathogenesis of cGVHD $^{9-11}$. CXCR3 is a Th1-associated inflammatory chemokine receptor highly expressed on effector $\mathrm{T}$ cells, in particular Th1 cells ${ }^{12}$. CXCR3 ligands include interferon- $\gamma($ IFN- $\gamma$ )-inducible chemokines of the CXC family such as CXCL9, CXCL10, and CXCL11 ${ }^{13-15}$, as well as CXCL4 (PF4) and CXCL4V1 (PF4V1) ${ }^{16,17}$. CXCR3 ${ }^{+}$Th1 can increase local secretion of IFN- $\gamma$ in inflamed tissues, which in turn stimulates CXCR3 ligand expression. In pathological conditions, the interplay between CXCR3 pathway and IFN- $\gamma$ can lead to excessive immune responses and amplified inflammation ${ }^{18}$. CXCR3 overexpression was also found in a $\mathrm{B}$ cell subset that is expanded in cGVHD patients ${ }^{19}$. Furthermore, the CXCR3 
axis has been shown to regulate endothelial cell apoptotic death, while endothelial cell alteration is a typical finding in cGVHD $^{20,21}$. Increased expression of CXCR3 ligands was found in both target tissues and the circulatory system in cGVHD ${ }^{22-27}$. Thus, CXCR3 ligands are potentially important mediators of cGVHD.

In the present study, we investigated the interplay of serum CXCR3 ligand concentrations and selected polymorphisms with regard to the incidence of severe cGVHD after alloSCT.

\section{Patients and methods \\ Patient cohorts}

Patients were recruited from two independent transplant centers and divided into three cohorts, including the training cohort (without statin-based endothelial protection (SEP), Heidelberg), SEP cohort (Heidelberg), and validation cohort (no SEP, Berlin). Patients were eligible if they had been transplanted between 2002 and 2014 in one of the two institutions, survived at least 6 months after alloSCT, and had biological material as needed for this study available. Human leukocyte antigen (HLA)-identical related donors were chosen if an HLA-identical (10/10) unrelated donor was not available or if an HLA-matched unrelated donor $(9 / 10)$ was not available. SEP consists of pravastatin $20 \mathrm{mg} /$ day and ursodeoxycholic acid (UDCA) $3 \times 250 \mathrm{mg} / \mathrm{day}^{28,29}$, and was used as a standard treatment in Heidelberg University Hospital since 01/2010. Written informed consent according to the Declaration of Helsinki was obtained from all patients, and the local Ethics committees had approved sample and data collection.

\section{GVHD prophylaxis and cGVHD diagnosis and grading}

GVHD prophylaxis and treatment were performed as previously described ${ }^{30}$. Severe cGVHD was defined as previously described according to the NIH consensus criteria $^{7}$, namely involvement of at least one organ system in its severest form (corresponding to being awarded 3 points in the NIH grading system, with the exception of lung cGVHD for which 2 points are sufficient). Nonsevere cGVHD was defined as any cGVHD not fulfilling the criteria of severe $\mathrm{cGVHD}^{8}$.

\section{Single-nucleotide polymorphism (SNP) analyses}

A total of 18 polymorphisms from CXCL4, CXCL4V1, CXCL9-11, and CXCR3 genes were identified by tagging approach using 1000 Genomes database for the Caucasian population. For CXCL4 and CXCL4V1 locus, a region of 152 kb (hg19: Chr4: 74,708,750-74,861,174) was screened and seven SNPs (rs409336, rs6810940, rs655328, rs28472816, rs3097412, rs1429638, and rs17811212; Supplemental Fig. 1) with minor allele frequency $\geq 0.05$ that tagged an additional 147 SNPs $\left(r^{2} \geq 0.8,71 \%\right.$ of SNPs in the region) were selected. For CXCL9-11 locus, a region of
200 kb (hg19: Chr4: 76,842,428-77,042,568) was screened and seven SNPs (rs8878, rs884304, rs3733236, rs2276885, rs6849878, rs67413521, and rs4282209; Supplemental Fig. 1) with minor allele frequency $\geq 0.05$ that tagged an additional 456 SNPs $\left(r^{2} \geq 0.8,76 \%\right.$ of SNPs in the region) were selected. For CXCR3 gene locus, a region of $43 \mathrm{~kb}$ (hg19: ChrX: $70,815,766-70,858,367)$ was screened and four SNPs (rs2280964, rs3091304, rs6625809, and rs3091305; Supplemental Fig. 1) with minor allele frequency $\geq 0.05$ that tagged an additional two SNPs $\left(r^{2} \geq 0.8\right)$ in the region were selected for genotyping. Genomic DNA from patients was genotyped using an allele-specific method (TaqMan Technology, Applied Biosystems, Foster City, CA). For quality control purpose, $10 \%$ of the samples was blindly replicated. PCR plates were read on a ViiA7 real-time instrument (Applied Biosystems) and QuantStudio Real-Time PCR Software was used to call genotypes.

\section{Serological analyses}

Prospectively collected serum samples taken once weekly before alloSCT until day +28 were analyzed for CXCL9 serum levels. Kinetics of CXCL9 levels were assessed in the context of calcineurin inhibitors (CNIs). For this purpose, a slope (differences/time) was calculated representing the recovery rate of serum CXCL9 until day +28 (Slope CXCL9 $=\frac{\text { CXCL9 } 9_{\text {day } 28}-\text { Lowest CXCL }_{\text {days } 0-14}}{28-\text { day }}$ ). Serum CXCL10 and CXCL11 levels were analyzed before transplant and on day +28 . Endothelial markers angiopoietin-2 and $\mathrm{sCD} 141$ on day +28 were measured by ELISA (R\&D, UK).

\section{Dual-luciferase reporter assays}

The CXCL9 promoter region with $6.8 \mathrm{~kb}$ (hg19, Chr4: $76,928,615-76,935,456)$ upstream of the start codon was cloned into pGL4.10 [luc2] promoter-less vector ("wildtype construct"). In addition, we generated four reporter constructs with QuikChange Site-Directed Mutagenesis Kit (Invitrogen). Three constructs carried variant alleles for rs884304 and two tagged SNPs (rs884004 and rs2869462) and one construct carried variant alleles for all the three SNPs. All five constructs were confirmed by Sanger sequencing. The constructs were transiently transfected into HEK293T cells together with Renilla luciferase vector as control using Lipofectamine 2000 reagent (Invitrogen) and incubated for $24 \mathrm{~h}$ without treatment or treated with IFN- $\gamma(300 \mathrm{U} / \mathrm{mL})$ and/or cyclosporin A (CsA, $1 \mu \mathrm{M})$ or FK506 (tacrolimus, $1 \mu \mathrm{M})$. Cells were subsequently lysed and assayed for luciferase activity. The effect of CNIs on CXCL9 promoter activity of the constructs was calculated as:

Effect of CNIs $=1-\frac{\text { Luciferase activity with CNIs }}{\text { Luciferase activity without CNIs }}$ 


\section{Statistical analysis}

Differences in categorical clinical covariates in patients were analyzed by Fisher's exact test or $X^{2}$ test. Mann-Whitney $U$ test was used for comparisons of quantitative continuous variables. Genotype frequencies of SNPs were tested in subjects for deviation from Hardy-Weinberg equilibrium. Median follow-up time was estimated by reverse Kaplan-Meier estimator. CXCL9-11 serum levels were $\log 2$-transformed because the distribution was highly left-skewed. Cause-specific Cox regression was used in univariate analysis, including CXCL9 serum levels as a continuous variable. The effect of the polymorphisms, using additive, dominant, and recessive models, was evaluated with regard to the risk of severe CGVHD, which was calculated from the date of alloSCT to the day of developing severe cGVHD. Relapse before severe cGVHD and nonrelapse death without severe cGVHD were considered as competing events for severe cGVHD. To account for the competing risks, cumulative incidence function was implemented, and statistical significance was determined using the Gray's $K$-sample test.

Severe cGVHD, relapse before severe cGVHD and NRM were treated as competing events and analyzed by cause-specific Cox regression models. Covariates included in multivariable models were age, diagnosis (lymphoid vs. myeloid), matched vs. mismatched donor, sex of donor and recipient, and usage of antithymocyte globulin (ATG). Prediction error curves and concordance index curves were generated to assess the model performance.

All statistical tests were two-sided. Hazard ratio (HR) was estimated with $95 \%$ confidence intervals (CIs). Values of $P$ $<0.05$ were considered statistically significant. All statistical analyses were carried out using statistical software $\mathrm{R}$ (version 3.5.2) together with the $\mathrm{R}$ packages dplyr (0.8.3), survival (2.44-1.1), cmprsk (2.2-8), and pec (2018.07.26).

\section{Results}

\section{Patient characteristics}

Overall, 688 patients (training cohort: $n=287$; SEP cohort: $n=401$ ) from Heidelberg University Hospital fulfilled the eligibility criteria for this study (Supplemental Fig. 2). Of these, 545 patients (no SEP: $n=242$; SEP: $n=303$ ) with available DNA were successfully genotyped for 4 CXCR3 and 14 CXCL4/4V/9/-10/-11 polymorphisms. Two hundred and two patients from Charite (Berlin) qualified for the validation cohort. Baseline and transplant-associated characteristics are shown in Table 1 and Supplemental Table 1. Overall, 50 of 287 patients (17\%) in the training cohort, 53 of 401 patients $(13 \%)$ in the SEP cohort, and 48 of 202 patients $(24 \%)$ in the validation cohort developed at least one episode of severe GVHD after a median time of $12.9,12.5$, and 13.8 months, respectively. The estimated median follow-up time was 72.5 months (95\% CI 67.1-77.3) for patients in Heidelberg
Table 1 Patient characteristics in the training cohort, validation cohort, and SEP cohort.

\begin{tabular}{|c|c|c|c|}
\hline & $\begin{array}{l}\text { Training } \\
\text { cohort no SEP, } \\
n=287\end{array}$ & $\begin{array}{l}\text { Validation } \\
\text { cohort no } \\
\text { SEP, } n=202\end{array}$ & $\begin{array}{l}\text { SEP cohort, } \\
n=401\end{array}$ \\
\hline $\begin{array}{l}\text { Median age at } \\
\text { alloSCT (years, range) }\end{array}$ & $50(17-71)$ & $50(19-72)$ & $57(19-76)$ \\
\hline \multicolumn{4}{|l|}{$\operatorname{Sex}(n, \%)$} \\
\hline Female & $114(40)$ & $72(36)$ & $161(40)$ \\
\hline Male & $173(60)$ & $130(64)$ & $240(60)$ \\
\hline \multicolumn{4}{|l|}{ Donor } \\
\hline $\mathrm{RD}$ & $108(38)$ & $65(32)$ & $104(26)$ \\
\hline UD & $179(62)$ & $137(68)$ & $297(74)$ \\
\hline \multicolumn{4}{|c|}{ Donor-recipient HLA matching } \\
\hline Matched donor & $214(75)$ & $195(97)$ & $327(82)$ \\
\hline Mismatched dono & $73(25)$ & $7(3)$ & $74(18)$ \\
\hline \multicolumn{4}{|c|}{ Sex mismatch (donor-recipient) $(n, \%)$} \\
\hline $\begin{array}{l}\text { Male-male, } \\
\text { Female-Female }\end{array}$ & $164(57)$ & $109(54)$ & $222(55)$ \\
\hline Male-female & $71(24)$ & $45(22)$ & $103(26)$ \\
\hline Female-male & $52(19)$ & $48(24)$ & $76(19)$ \\
\hline \multicolumn{4}{|l|}{ Disease $(n, \%)$} \\
\hline AML & $76(26)$ & $83(41)$ & $146(36)$ \\
\hline MDS, MPN, AA & $45(16)$ & $44(22)$ & $72(18)$ \\
\hline $\begin{array}{l}\text { Lymphoma, } \\
\text { ALL, CLL }\end{array}$ & $111(39)$ & $61(30)$ & $141(35)$ \\
\hline MM, Amyloidosis & $55(19)$ & $14(7)$ & $42(11)$ \\
\hline \multicolumn{4}{|c|}{ Disease score before alloSCT $(n, \%)$} \\
\hline 0 & $99(34)$ & $92(46)$ & $138(34)$ \\
\hline 1 & $41(15)$ & $80(40)$ & $147(37)$ \\
\hline 2 & $134(47)$ & $25(12)$ & $108(27)$ \\
\hline NA & $13(5)$ & $5(2)$ & $8(2)$ \\
\hline \multicolumn{4}{|l|}{ Stem cell source $(n, \%)$} \\
\hline Peripheral stem cells & $272(95)$ & $200(99)$ & $382(95)$ \\
\hline $\begin{array}{l}\text { Bone marrow } \\
\text { stem cells }\end{array}$ & $15(8)$ & $2(1)$ & $19(5)$ \\
\hline \multicolumn{4}{|l|}{ Conditioning $(n, \%)$} \\
\hline MAC & $51(18)$ & $82(41)$ & $15(4)$ \\
\hline APL & $7(2)$ & & $46(11)$ \\
\hline $\mathrm{RIC}$ & $229(80)$ & $120(59)$ & $340(85)$ \\
\hline \multicolumn{4}{|l|}{$\operatorname{ATG}(n, \%)$} \\
\hline No & $140(49)$ & $81(40)$ & $103(26)$ \\
\hline Yes & $147(51)$ & $121(60)$ & $298(74)$ \\
\hline
\end{tabular}


Table 1 continued

\begin{tabular}{|c|c|c|c|}
\hline & $\begin{array}{l}\text { Training } \\
\text { cohort no SEP, } \\
n=287\end{array}$ & $\begin{array}{l}\text { Validation } \\
\text { cohort no } \\
\text { SEP, } n=202\end{array}$ & $\begin{array}{l}\text { SEP cohort, } \\
n=401\end{array}$ \\
\hline \multicolumn{4}{|c|}{ Combined genetic score $(n, \%)$} \\
\hline Low-risk & $190(66)$ & $160(79)$ & $235(59)$ \\
\hline High risk & $52(18)$ & $42(21)$ & $68(17)$ \\
\hline NA (no DNA) & $45(16)$ & & $98(24)$ \\
\hline $\begin{array}{l}\text { CXCL9 } \text { pre }^{a} \text { median } \\
(\mathrm{pg} / \mathrm{mL}, \mathrm{IQR})\end{array}$ & $\begin{array}{l}n=109 \\
204.5 \\
(108.7-629.5)\end{array}$ & & $\begin{array}{l}n=296 \\
175.7(72.3- \\
542.1)\end{array}$ \\
\hline $\begin{array}{l}\text { CXCL9 day } 28+^{\mathrm{b}} \\
\text { median }(\mathrm{pg} / \mathrm{mL}, \mathrm{IQR})\end{array}$ & $\begin{array}{l}n=152 \\
315.3 \\
(141.2-673.3)\end{array}$ & & $\begin{array}{l}n=342 \\
295.5(114.8- \\
598.8)\end{array}$ \\
\hline \multicolumn{4}{|c|}{ CXCL10 pre median (pg/mL, IQR) } \\
\hline $\begin{array}{l}\text { CXCL10 day } 28+ \\
\text { median (pg/mL, IQR) }\end{array}$ & $\begin{array}{l}n=146 \\
152.9 \\
(65.6-283.7)\end{array}$ & & $\begin{array}{l}n=333 \\
128.7(56.7- \\
242.0)\end{array}$ \\
\hline $\begin{array}{l}\text { CXCL11 pre median } \\
(\mathrm{pg} / \mathrm{mL}, \mathrm{IQR})\end{array}$ & $\begin{array}{l}n=103 \\
79.8 \\
(24.3-183.4)\end{array}$ & & $\begin{array}{l}n=289 \\
70.4(24.5- \\
165.0)\end{array}$ \\
\hline $\begin{array}{l}\text { CXCL11 day } 28+ \\
\text { median (pg/mL, IQR) }\end{array}$ & $\begin{array}{l}n=146 \\
119.2 \\
(61.2-211.8)\end{array}$ & & $\begin{array}{l}n=333 \\
122.1(53.5- \\
204.6)\end{array}$ \\
\hline
\end{tabular}

SEP statin-based endothelial prophylaxis, $A A$ aplastic anemia, ALL acute lymphoblastic leukemia, alloSCT allogeneic stem cell transplantation, $A M L$ acute myelogenous leukemia, ATG antithymocyte globulin, CLL chronic lymphocytic leukemia, disease score: $0=C R 1,1=C R 2,2=$ all other, $M A C$ myeloablative conditioning, MPS myeloproliferative syndrome, MM multiple myeloma, $R D$ related donor, RIC reduced intensity conditioning, UD unrelated donor. ${ }^{\text {a }}$ Measured pre-transplant.

${ }^{\mathrm{b}}$ Measured at day 28 post transplant.

center and 66.7 months (95\% CI 60.6-77.3) for Berlin cohort.

\section{Identification of high-risk genotypes of SNPs from CXCR3} and ligand genes (CXCL4/9-11) in the training cohort

To determine the effect of polymorphisms on severe cGVHD, the polymorphisms were analyzed under the additive, dominant, and recessive models; the most significant statistic among the three models is considered to be an indicator of association between the polymorphism and the outcome (Supplemental Table 2). Out of the 18 polymorphisms analyzed, rs884304 in CXCL9 locus showed statistically significant association $(P=0.010)$ with a risk of severe cGVHD in the dominant model. Patients who were carriers of the variant allele (AA/AG genotypes) of rs884304 showed significantly increased risk for severe cGVHD (HR 2.35, 95\% CI 1.23-4.51) compared to patients with homozygote GG genotype (Fig. 1A). The other polymorphisms did not show any isolated statistically significant association with severe cGVHD in the training cohort.

\section{Selection of polymorphisms for computing genetic risk group}

Two genetic risk groups A and B were built based on statistically significant polymorphism rs884304 and three other polymorphisms (rs3733236 (CXCL9-11), rs4282209 (CXCL9-11), rs655328 (CXCL4V1)) that showed a Gray's test $P$ value of $<0.10$ with a risk of severe cGVHD (Supplemental Fig. 3).

For genetic risk group A, patients with high-risk genotypes (AA/AG) for rs884304 were marked as high-risk group, others (GG) were considered as low-risk group. For genetic risk group B, patients with rs3733236GG, rs4282209GG, and rs655328CC/CT at the same time were considered as high-risk group, others were classified as low-risk group. Besides genetic risk group A, genetic risk group $B$ is also significantly associated with the risk of severe cGVHD (Fig. 1B, $P=0.005$ ). If the two genetic risk groups A and B were assessed together, patients who were at high risk for both genetic risk groups were associated with the highest risk of severe cGVHD (Fig. 1C). Therefore, a combined genetic risk group was established for CXCR3 ligand genes (CXCL4 and CXCL9-11). Patients with any low-risk genotypes for the two genetic risk groups (rs884304GG and three SNPs (rs3733236AA/AG, rs4282209AA/AG, and rs655328TT)) were classified into the low-risk group, patients with all high-risk genotypes (rs884304AA/AG, rs3733236GG, rs4282209GG, and rs655328CC/CT) were classified into high-risk group.

\section{Association of CXCL9 serum levels with risk of severe cGVHD}

We previously demonstrated that CXCL9 levels correlate with the risk of developing severe cGVHD when measured at the onset of mild symptoms ${ }^{8}$. In the present study, we analyzed CXCL9, CXCL10, and CXCL11 serum levels before and on day +28 after alloSCT in the training cohort. In four increasing intervals (Q1, Q2, Q3, and Q4) separated by quartiles of CXCL9, patients in Q4 were associated with increased risk for severe cGVHD in the training cohort (Fig. 1D, Q4 vs. Q1-3, HR 2.93, 95\% CI 1.34-6.43, $P=0.007)$. In univariable analysis, day +28 serum CXCL9 levels were significantly associated with risk of severe cGVHD in the training cohort (HR for a twofold change $1.38,95 \%$ CI $1.10-1.73, P=0.006)$.

No significant association was found for either CXCL10 $(P=0.176)$ or CXCL11 $(P=0.524)$ day +28 serum levels with risk of severe cGVHD. Similarly, serum levels of CXCL9, 10, and 11 taken prior to conditioning therapy did not correlate with severe cGVHD $(P=0.777,0.805$, and 0.346 , respectively). 
A

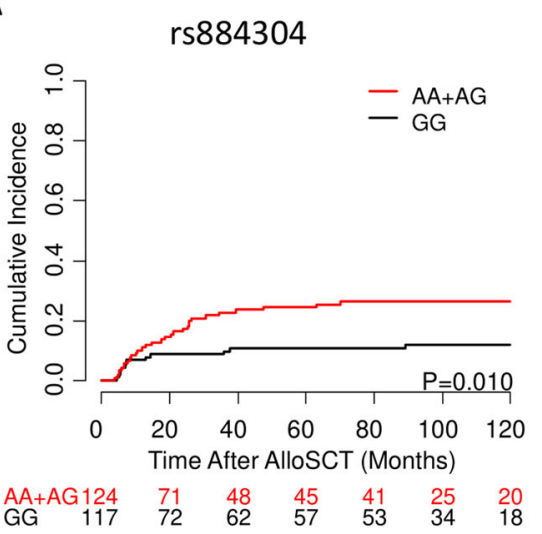

C

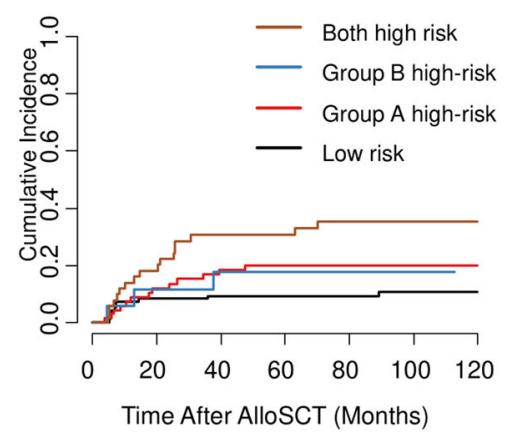

B

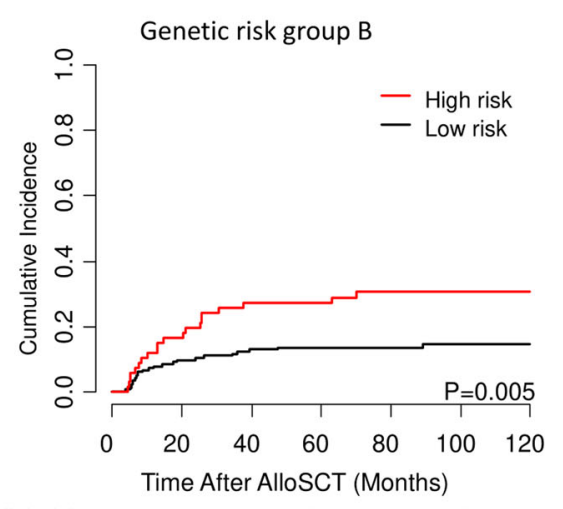

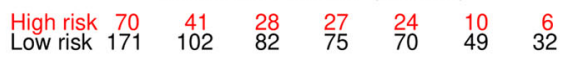
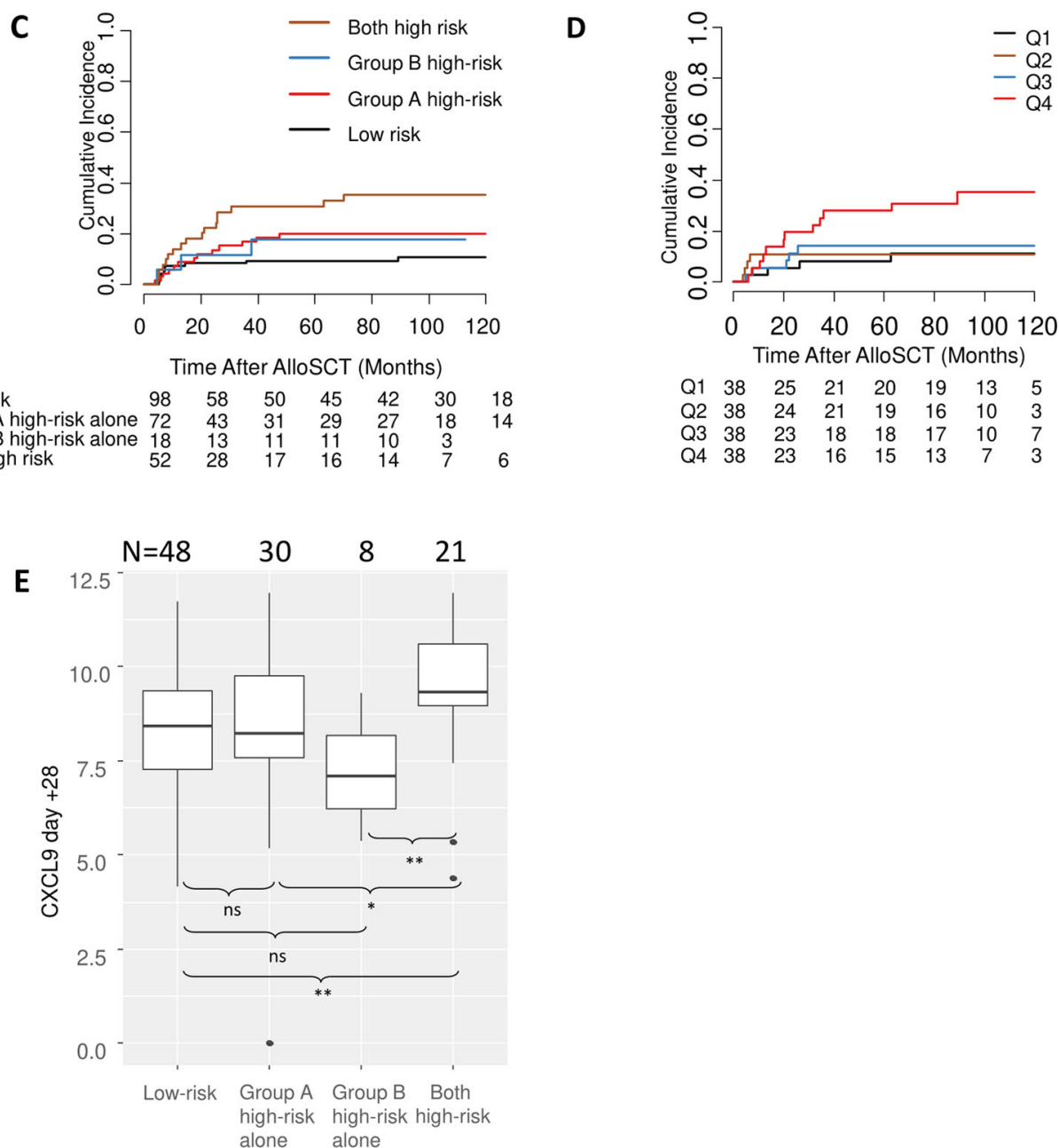

Fig. 1 Association of single-nucleotide polymorphisms (SNPs) in CXCR3 ligand genes and serum chemokines with the incidence of severe chronic graft-versus-host disease (GVHD). A Carriers of the variant allele for CXCL9 polymorphism rs884304 (AA + AG) were associated with a significantly higher incidence of severe chronic GVHD in the training cohort. B Effects of the three candidate SNPs for genetic risk group B on the incidence of severe chronic GVHD after alloSCT. Genetic risk group B was based on rs3733236, rs4282209, and rs655328. C Association of severe chronic GVHD incidence with genetic risk groups A and B. D Association of serum CXCL9 levels with a cumulative incidence of severe chronic GVHD after alloSCT in the training cohort. E Association of day +28 CXCL9 serum levels with genetic risk groups $A$ and B. n.s. not significant; ${ }^{*} P \leq 0.05 ;{ }^{* *} P<$ $0.01 ;{ }^{* * *} P<0.001$. 
Comparing the genotype data with serum CXCL9 levels, patients in the combined genetic high-risk group had statistically significantly higher serum CXCL9 levels on day +28 post alloSCT (Fig. 1E).

\section{Combined genetic risk in the training and validation cohorts}

Taken together, high-risk polymorphisms were found in $21 \%(52 / 242)$ patients in the training cohort and $20 \%(40 /$ 202) patients in the validation cohort (Table 1). The highrisk genotype was significantly associated with an increased risk of severe cGVHD in both the training and validation cohort (Fig. 2A).

Multivariable analyses to assess the influence of highrisk genotypes on outcomes after alloSCT were performed with covariates age, diagnosis (lymphoid vs. myeloid), matched vs. mismatched donor, sex of donor and recipient, and usage of ATG. As this model only comprised pre-transplantation factors, serum levels of day +28 CXCL9 were not included. Results are shown in Tables 2 and 3. For severe cGVHD, similar HRs for the high-risk group were observed in the training (HR 2.48, 95\% CI 1.33-4.64, $P=0.004$ ) and the validation cohort (HR 2.95, 95\% CI 1.56-5.58, $P=0.001$ ).

The result of the training cohort was validated using a cause-specific Cox model fitted to the validation cohort with an offset equal to the effect of the genetic risk group in the training cohort. The re-estimated effect of genetic risk group in the validation cohort was not significantly different from 1 (HR 1.19, 95\% CI 0.63-2.24, $P=0.60$ ). This means the effect of genetic risk group in the validation cohort was not significantly different from the effect in the training set. The effect was also validated using prediction error curves. A detailed validation method is provided in Supplemental statistical methods (Supplemental Fig. 4).

The predictive power of the genetic risk for severe cGVHD was assessed using prediction error and concordance index over time of two different cause-specific Cox models. The reference models included only the clinical parameters: age, diagnosis (lymphoid vs. myeloid), matched vs. mismatched donor, sex of donor and recipient, and usage of ATG. CXCL models included the genetic risk group (high-risk vs. low-risk) predictor in addition to the same clinical parameters. Lower prediction error and higher concordance index indicate better performance of a model. The model with the genetic risk group predictor obtained significantly lower prediction error and significantly higher concordance index over time in both training and validation cohort (Supplemental Fig. 5).

\section{Loss of predictive impact of the genetic risk group in the context of SEP}

Patients receiving SEP were consecutively recruited upon standard policy change from $01 / 2010$ in the
Heidelberg center. In order to control for random effects due to alterations in clinical practice over time, patients with SEP were divided into two separate cohorts (patients transplanted before 12/2011, high risk: $n=31$, low risk: $n=113 ; 01 / 2012-06 / 2014$, high risk: $n=37$, low risk: $n=122)$. The introduction of SEP abolished the impact of high-risk genotypes in both groups of patients (Fig. 2B). The protective effect of SEP was restricted to the high-risk group and the risk of severe cGVHD was reduced to the level of the low-risk group. The high-risk group also did not show statistically significant effects in the multivariable analysis (Supplemental Table 3; HR 1.48, 95\% CI $0.68-3.21, P=0.321)$. In addition, SEP was associated with significantly reduced serum CXCL9 levels at day +28 . Again, the effect was confined to the genetic highrisk group (Fig. 2C).

\section{ATG reduced severe CGVHD risk irrespective of genetic risk}

We investigated the effect of ATG on severe cGVHD and interaction with genetic risk groups. Patients receiving ATG had a significantly lower risk of developing severe cGVHD in both training and validation cohorts. Unlike SEP, the effect of ATG did not differ between genetic high- and low-risk groups of CXCR3 ligand genes (Supplemental Fig. 6), and within the ATG group, genetic high-risk patients still had an increased risk of severe cGVHD when compared to low-risk patients (combined training and validation cohorts, $n=474, \mathrm{HR} 2.04,95 \% \mathrm{CI}$ 1.15-3.60, $P=0.015)$. In addition, ATG usage did not alter serum CXCL9 levels at day +28 . In the high-risk group, SEP could fully substitute the protective effect of ATG, but there were no additive effects in patients receiving both. In contrast, ATG was effective independent of SEP in the low-risk group (Supplemental Fig. 7).

\section{The association of the combined genetic risk with acute GVHD}

A prior episode of acute GVHD is a risk factor for cGVHD. We investigated the association of the high-risk genotype with acute GVHD and NRM after GVHD in the complete cohort of patients (including deaths before 6 months, Supplemental Table 4). No significant association was found for genetic high risk in the CXCR3 ligand genes with the incidence of acute GVHD or grade 3-4 GVHD, nor with NRM after GVHD (Supplemental Table 5).

\section{Functional evaluation of CXCL9 polymorphisms using dual- luciferase assays}

We hypothesized that CXCL9 promoter activity is regulated by the polymorphism rs884304. Indeed, rs884304AA/AG correlated with increased CXCL9 serum levels on day +28 (Fig. 3A). We performed luciferase reporter assays in the presence and absence of IFN- $\gamma$ and 
A

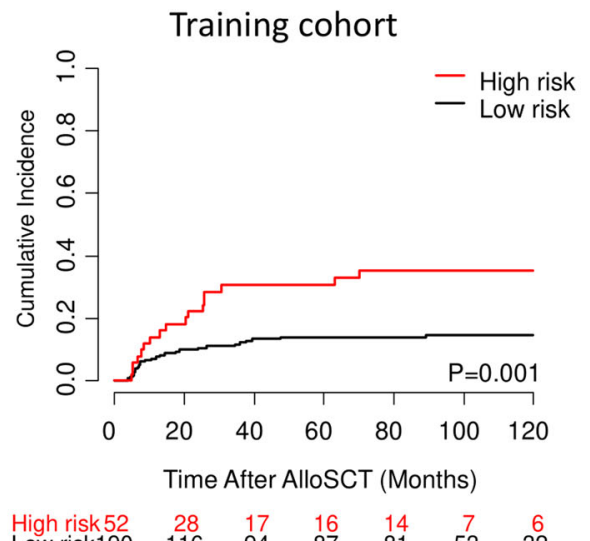

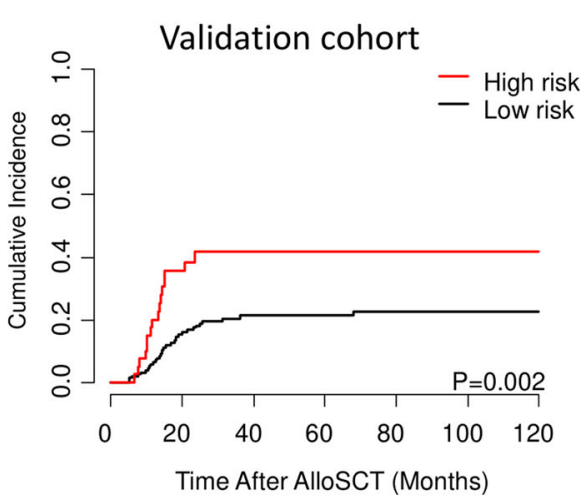

$\begin{array}{lrrrrrr}\text { High risk 40 } & 16 & 9 & 5 & 3 & 1 & 1 \\ \text { Low risk162 } & 79 & 49 & 37 & 20 & 11 & 6\end{array}$
B

Before 2012

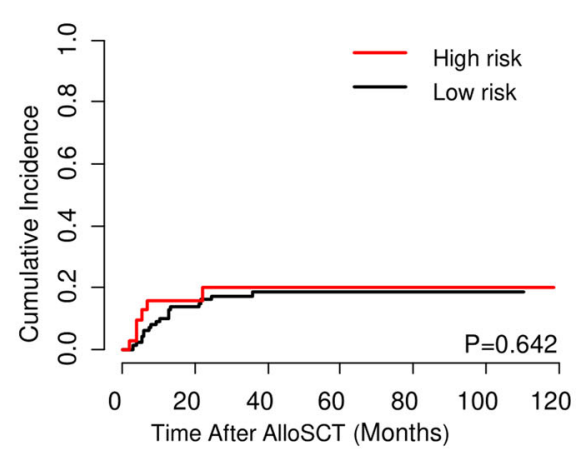

$\begin{array}{llrrrr}\text { High risk } 31 & 20 & 15 & 9 & 1 & 1 \\ \text { Low risk 113 } & 76 & 67 & 47 & 6 & 2\end{array}$ after 2012

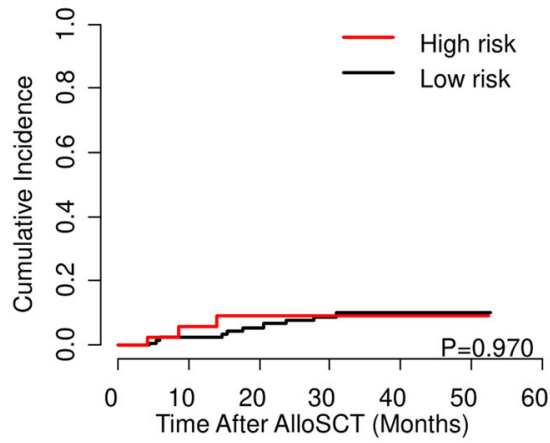

$\begin{array}{lllllll}\text { High risk } & 37 & 28 & 25 & 22 & 12 & 2\end{array}$

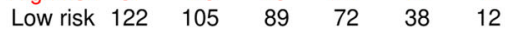

$N=8621 \quad 19252$

C

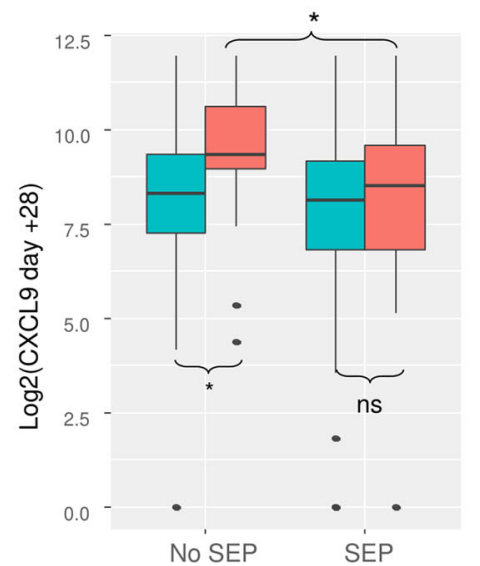

Low-risk

High-risk

Fig. 2 The effect of genetic risk (CXCR3 ligands) on severe chronic graft-versus-host disease (GVHD). A Association of the combined genetic risk group with the incidence of severe chronic GVHD in the training cohort and validation cohort. B No difference could be observed between the genetic risk groups in patients with SEP in two consecutive cohorts ( $n=144$ and 159, respectively). C Association of serum CXCL9 levels at day +28 with the combined genetic risk group in patients without and with SEP. n.S. not significant; ${ }^{*} P \leq 0.05$. 
Table 2 Multivariable analysis of the incidence of severe cGVHD, OS, NRM, and relapse in the training cohort.

\begin{tabular}{|c|c|c|c|c|c|c|c|c|c|c|c|c|}
\hline \multirow[t]{2}{*}{ Covariates } & \multicolumn{4}{|c|}{ Severe chronic GVHDa } & \multicolumn{4}{|l|}{ OS } & \multicolumn{2}{|l|}{$\mathrm{NRM}^{\mathrm{a}}$} & \multicolumn{2}{|l|}{ Relapse $^{a}$} \\
\hline & $N$ & ScGVHD & $\mathrm{HR}(95 \% \mathrm{Cl})$ & $P$ & $N$ & death & $\mathrm{HR}(95 \% \mathrm{Cl})$ & $P$ & HR $(95 \% \mathrm{Cl})$ & $P$ & HR $(95 \% \mathrm{Cl})$ & $P$ \\
\hline \multicolumn{13}{|l|}{ Genetic risk } \\
\hline Low-risk group & 190 & 26 & Ref. & & 190 & 97 & Ref. & & Ref. & & Ref. & \\
\hline High-risk group & 52 & 17 & $2.48(1.33-4.64)$ & 0.004 & 52 & 27 & $1.09(0.70-1.69)$ & 0.697 & $1.34(0.68-2.65)$ & 0.402 & $0.90(0.52-1.57)$ & 0.710 \\
\hline Age & 242 & 43 & $1.01(0.98-1.03)$ & 0.622 & 242 & 124 & $1.02(1.00-1.03)$ & 0.041 & $1.04(1.01-1.07)$ & 0.006 & $1.00(0.98-1.02)$ & 0.754 \\
\hline \multicolumn{13}{|l|}{ Recipient sex } \\
\hline Female & 93 & 19 & Ref. & & 93 & 48 & Ref. & & Ref. & & Ref. & \\
\hline Male & 149 & 24 & $0.88(0.47-1.64)$ & 0.694 & 149 & 76 & $1.00(0.69-1.45)$ & 0.979 & $1.52(0.80-2.90)$ & 0.199 & $0.77(0.49-1.21)$ & 0.252 \\
\hline \multicolumn{13}{|l|}{ Donor sex } \\
\hline Female & 74 & 21 & Ref. & & 74 & 38 & Ref. & & Ref. & & Ref. & \\
\hline Male & 168 & 22 & $0.5(0.29-0.98)$ & 0.042 & 168 & 86 & $1.07(0.72-1.58)$ & 0.741 & $0.95(0.50-1.78)$ & 0.863 & $0.93(0.57-1.49)$ & 0.755 \\
\hline \multicolumn{13}{|l|}{ Donor } \\
\hline Matched donor & 179 & 34 & Ref. & & 179 & 91 & Ref. & & Ref. & & Ref. & \\
\hline Mismatched donor & 63 & 9 & $1.82(0.76-4.08)$ & 0.180 & 63 & 33 & $1.45(0.91-2.32)$ & 0.115 & $2.33(1.08-5.03)$ & 0.032 & $0.92(0.51-1.66)$ & 0.772 \\
\hline \multicolumn{13}{|l|}{ Disease type } \\
\hline Myeloid $^{\mathrm{b}}$ & 102 & 20 & Ref. & & 102 & 47 & Ref. & & Ref. & & Ref. & \\
\hline Lymphoid $^{c}$ & 140 & 23 & $1.05(0.57-1.95)$ & 0.869 & 140 & 77 & $1.25(0.86-1.82)$ & 0.238 & $0.78(0.43-1.43)$ & 0.419 & $2.26(1.38-3.71)$ & 0.001 \\
\hline \multicolumn{13}{|l|}{ ATG } \\
\hline No & 117 & 32 & Ref. & & 117 & 68 & Ref. & & Ref. & & Ref. & \\
\hline Yes & 125 & 11 & $0.26(0.11-0.59)$ & 0.001 & 125 & 56 & $0.68(0.45-1.03)$ & 0.067 & $0.49(0.24-1.02)$ & 0.057 & $0.86(0.52-1.42)$ & 0.559 \\
\hline
\end{tabular}

ATG antithymocyte globulin, $\mathrm{Cl}$ confidence interval, $H R$ hazard ratio, ScGVHD severe chronic graft-versus-host disease.

${ }^{a} \mathrm{HR}$ and $P$ value from cause-specific Cox models for competing risk. Death without ScGVHD was taken as the competing event for scGVHD. NRM and relapse were treated as competing events.

${ }^{\mathrm{b}}$ Myeloid: acute myeloid leukemia and myelodysplastic and myeloproliferative syndromes.

'Lymphoid: acute lymphoblastic leukemia, chronic lymphocytic leukemia, T/B cell lymphoma, and multiple myeloma.

CNIs to mimic the in vivo environment of post-alloSCT recipients.

All five constructs with the insert fragment possessed CXCL9 promoter activity and showed strong responses to IFN- $\gamma$ treatment. On average, a 9.7-fold increase of the luciferase activity was observed for the five constructs upon IFN- $\gamma$ activation, while the empty vector showed no response (Supplemental Fig. 8).

Upon IFN- $\gamma$ activation, significantly reduced suppressive effects of CsA on the luciferase activity $\left(\right.$ Effect of CsA $\left.=\left(1-\frac{\text { luci fearse activity with CsA }}{\text { luci fearse activity without CsA }}\right) * 100 \%\right)$ in the constructs carrying variant alleles of rs884304 $(P<$ $0.001)$, rs884004 $(P<0.001)$, and the construct with all three SNPs $(P=0.001)$ were observed compared to the wild-type construct, while the construct carrying the variant allele of rs2869462 showed no significant difference (Fig. 3B). Similar effects of the rs884304 $(P<0.001)$ and rs884004 $(P<0.001)$ variant alleles were observed for FK506 (Fig. 3C).
CXCL9 serum levels decreased within 2 weeks after alloSCT and normalized until day +28 (Supplemental Fig. 9). CXCL9 serum level kinetics was modelled by slope calculation. Slope CXCL9 represents the CXCL9 recovery rate from the lowest point between days 0 and +14 until day +28 post transplant.

Patients carrying the variant allele A of rs884304 had a significantly steeper slope CXCL9 (Fig. 3D; $P=0.017$ ) in the training cohort. The steeper slopes suggest that CXCL9 levels increased more rapidly during hematological reconstitution in the context of CNI.

\section{Discussion}

GVHD remains a serious cause of morbidity and mortality in alloSCT. cGVHD is associated with poor quality of life, and prediction and prevention of cGVHD remain difficult. In the current study, we established a genetic risk group-based $C X C R 3$ ligand genes of the recipients as a novel predictor of severe cGVHD after alloSCT. Knowledge of the recipients' genetic risk could thus trigger 
Table 3 Multivariable analysis of the incidence of severe CGVHD, OS, NRM, and relapse in the validation cohort.

\begin{tabular}{|c|c|c|c|c|c|c|c|c|c|c|c|c|}
\hline \multirow[t]{2}{*}{ Covariates } & \multicolumn{4}{|c|}{ Severe chronic GVHD ${ }^{a}$} & \multicolumn{4}{|l|}{ OS } & \multicolumn{2}{|l|}{$\mathrm{NRM}^{\mathrm{a}}$} & \multicolumn{2}{|l|}{ Relapse $^{a}$} \\
\hline & $N$ & ScGVHD & HR $(95 \% \mathrm{Cl})$ & $P$ & $N$ & death & $\mathrm{HR}(95 \% \mathrm{Cl})$ & $P$ & HR $(95 \% \mathrm{Cl})$ & $P$ & $\mathrm{HR}(95 \% \mathrm{Cl})$ & $P$ \\
\hline \multicolumn{13}{|l|}{ Genetic risk } \\
\hline Low-risk group & 162 & 32 & Ref. & & 162 & 66 & Ref. & & Ref. & & Ref. & \\
\hline High-risk group & 40 & 16 & $2.95(1.56-5.58)$ & 0.001 & 40 & 17 & $1.16(0.67-2.00)$ & 0.590 & $1.74(0.68-4.43)$ & 0.248 & $0.78(0.42-1.44)$ & 0.423 \\
\hline Age & 202 & 48 & $1.00(0.98-1.02)$ & 0.970 & 202 & 83 & $1.01(0.99-1.03)$ & 0.278 & $1.03(0.99-1.06)$ & 0.176 & $1.01(0.99-1.03)$ & 0.331 \\
\hline \multicolumn{13}{|l|}{ Recipient sex } \\
\hline Female & 72 & 20 & Ref. & & 72 & 29 & Ref. & & Ref. & & Ref. & \\
\hline Male & 132 & 28 & $0.69(0.38-1.26)$ & 0.225 & 132 & 54 & $0.92(0.58-1.47)$ & 0.737 & $0.41(0.17-1.00)$ & 0.050 & $1.01(0.63-1.64)$ & 0.957 \\
\hline \multicolumn{13}{|l|}{ Donor sex } \\
\hline Female & 75 & 23 & Ref. & & 75 & 29 & Ref. & & Ref. & & Ref. & \\
\hline Male & 127 & 25 & $0.75(0.41-1.37)$ & 0.344 & 127 & 54 & $1.40(0.87-2.26)$ & 0.160 & $2.14(0.83-5.53)$ & 0.117 & $1.24(0.76-2.04)$ & 0.391 \\
\hline \multicolumn{13}{|l|}{ Donor } \\
\hline Matched donor & 195 & 47 & Ref. & & 195 & 79 & Ref. & & Ref. & & Ref. & \\
\hline Mismatched donor & 7 & 1 & $0.98(0.13-7.64)$ & 0.984 & 7 & 4 & $2.55(0.90-7.19)$ & 0.078 & $6.46(1.33-33.16)$ & 0.020 & $1.14(0.27-4.74)$ & 0.859 \\
\hline \multicolumn{13}{|l|}{ Disease type } \\
\hline Myeloid $^{\mathrm{b}}$ & 127 & 29 & Ref. & & 127 & 51 & Ref. & & Ref. & & Ref. & \\
\hline Lymphoid $^{c}$ & 75 & 19 & $0.77(0.41-1.44)$ & 0.416 & 75 & 32 & $1.08(0.67-1.73)$ & 0.762 & $2.10(0.84-5.28)$ & 0.115 & $1.05(0.64-1.74)$ & 0.842 \\
\hline \multicolumn{13}{|l|}{ ATG } \\
\hline No & 81 & 29 & Ref. & & 81 & 37 & Ref. & & Ref. & & Ref. & \\
\hline Yes & 121 & 19 & $0.41(0.22-0.76)$ & 0.005 & 121 & 46 & $0.88(0.55-1.41)$ & 0.590 & $0.66(0.26-1.64)$ & 0.367 & $1.00(0.61-1.65)$ & 0.988 \\
\hline
\end{tabular}

ATG antithymocyte globulin, $C l$ confidence interval, $H R$ hazard ratio, sCGVHD severe chronic graft-versus-host disease.

${ }^{a} \mathrm{HR}$ and $P$ value from cause-specific Cox models for competing risk. Death without ScGVHD was taken as the competing event for scGVHD. NRM and relapse were treated as competing events.

${ }^{b}$ Myeloid: acute myeloid leukemia and myelodysplastic and myeloproliferative syndromes.

'Lymphoid: acute lymphoblastic leukemia, chronic lymphocytic leukemia, T/B cell lymphoma, and multiple myeloma.

altered immunosuppressive or immunomodulating regimens, including ATG or SEP prophylaxis.

For cGVHD prediction, most studies focused on diagnostic and prognostic biomarkers at disease onset or in established cGVHD. A biomarker panel including CXCL9 at day +100 has been developed that can predict $\mathrm{cGVHD}^{31}$. However, the pathogenesis of cGVHD initiates earlier $^{32,33}$. In the present study, CXCL9 serum levels on day +28 were associated with the risk of severe cGVHD. These data suggest that immune-mediated pathways activated early after alloSCT may contribute to subsequent development of severe cGVHD.

In luciferase reporter assays, the variant allele of rs884304 and rs884004 showed resistance to the immunosuppressive effect of CNIs upon IFN- $\gamma$ activation. Calcineurin has been shown to interact with transcription factors including increasing NFIC transactivation and enhancing MEF2 DNA binding ${ }^{34-36}$. Chromatin immunoprecipitation-sequencing peaks of transcription factor binding sites for NIFC and MEF2A are found in the region containing the SNP rs884304 in ENCODE (Encyclopedia of DNA Elements) data ${ }^{37}$. It can be hypothesized that these enhancer risk variants can affect the binding of NIFC or MEF2, leading to less interaction with calcineurin, and thus reduced suppressive effects of CNIs. This may partly explain the effect of the CXCR3 ligands' SNPs on CXCL9 expression and severe cGVHD. The effects of the other three SNPs and how the combined effect alters CXCL9 expression need to be further investigated.

The CXCR3 signalling pathway could play a role in the pathogenesis of cGVHD by driving donor effector T cells into target tissues of the recipient ${ }^{38}$. Moreover, CXCR3 regulates apoptotic death of endothelial cells, and high concentrations of CXCL9 induced breakdown of the endothelial barrier through the CXCR3 pathway ${ }^{20,39}$. The interaction between the CXCR3 system and endothelial cells may facilitate the trafficking of donor $\mathrm{T}$ cells into target tissues and aggregate inflammation and tissue damage in cGVHD patients. This endothelial side of CXCR3 signalling may explain why SEP also reduced 
A

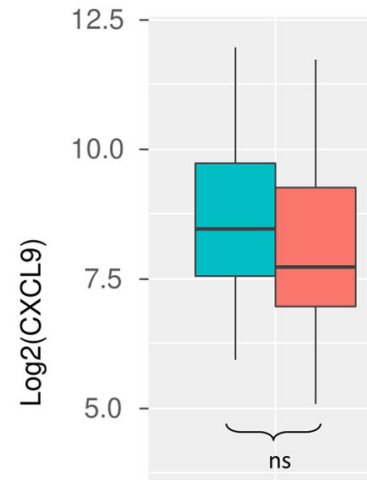

$2.5-$

$0.0-$ pre-transplant
6173

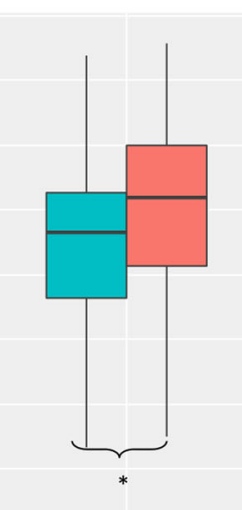

rs884304

苗 GG $A A+A G$
C

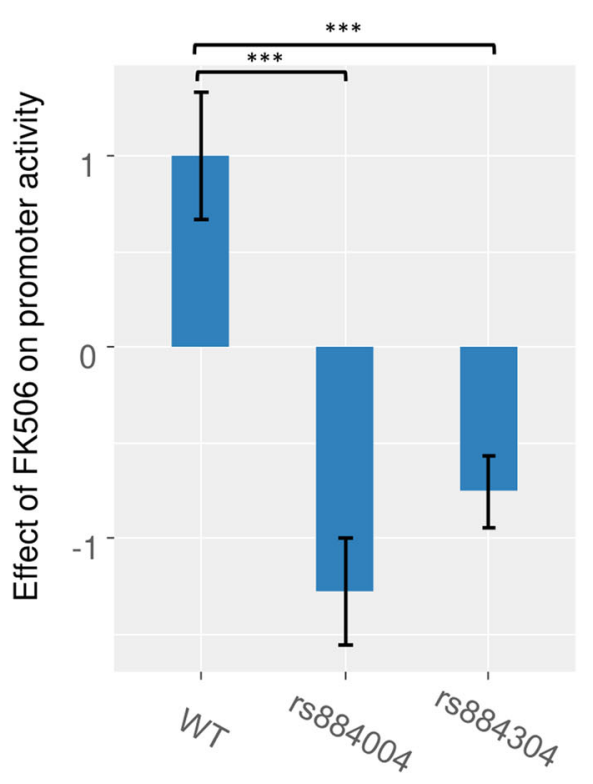

B

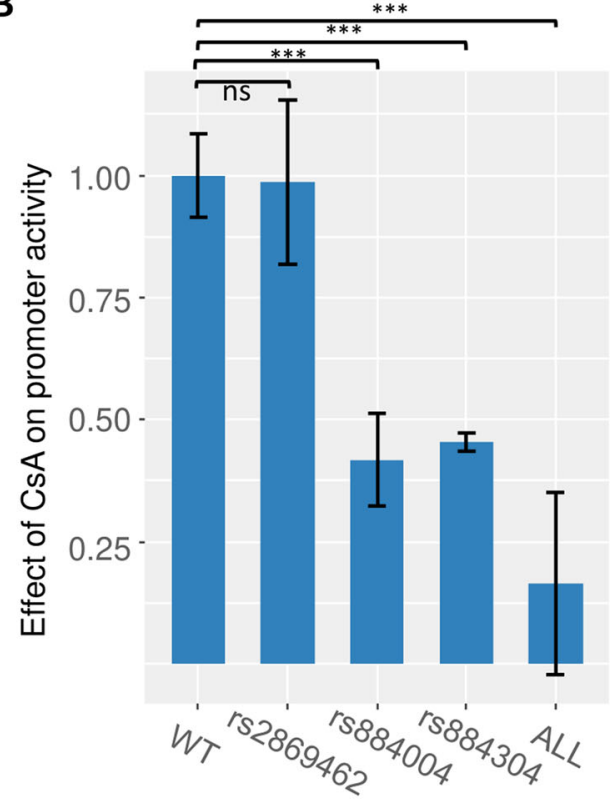

D $N=39$ $\mathrm{N}=42$

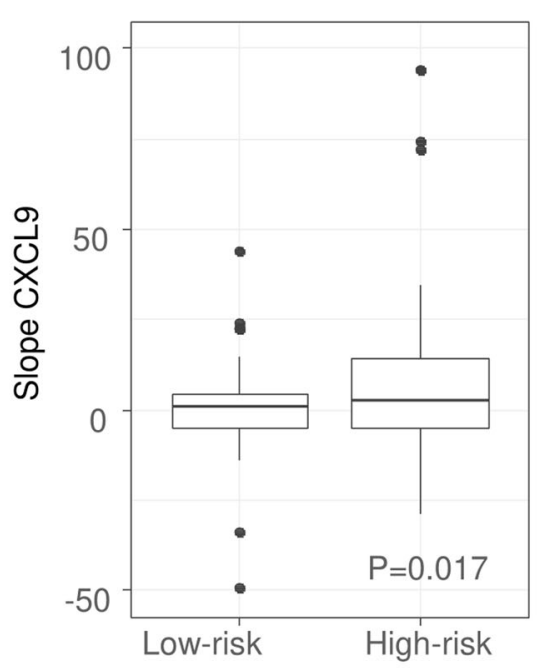

Fig. 3 The association of rs884304 with IFN- $\gamma$ induced promoter activity and CXCL9 recovery. A In patients, high-risk genotypes (AA + AG) of genetic risk group A (rs884304) were associated with significantly higher day +28 serum CXCL9 levels, but not with pre-transplant CXCL9. B In luciferase reporter assays, IFN- $\gamma$ activation revealed that the constructs carrying variant allele of rs884304 $(P<0.001)$, rs884004 $(P<0.001)$, and the one carrying variant alleles of all the three SNPs $(P=0.001)$, but not rs2869462, were associated with significantly reduced suppressive effect of CsA compared to the wild-type construct. The results were collected from three independent experiments. Luciferase activity was normalized to 1 relative to the WT and all data were plotted as the mean \pm SEM. C Similar to (B), with FK506 (tacrolimus) as calcineurin inhibitor. Results were collected from three independent experiments. Luciferase activity was normalized to 1 relative to the WT and all data were plotted as the mean \pm SEM. D Recovery rates of CXCL9 serum levels in patients from the lowest value post alloSCT until day $28+$ in the presence of calcineurin inhibitors (slope CXCL9) was higher in the genetic high-risk group. n.s. not significant; ${ }^{*} P \leq 0.05 ;{ }^{* *} P<0.001$. 
severe cGVHD in patients with already high CXCL9 levels on day +28 .

$\mathrm{B}$ cells are recognized as important players in the pathophysiology of $\mathrm{CGVHD}^{40,41}$. Notably, expression of CXCR3 is associated with aberrant B cell subpopulations reported in $\mathrm{CGVHD}^{19}$ and in other autoimmune diseases $^{42-44}$.

The SEP cohort in Heidelberg University Hospital showed a lower incidence of TAM, and reduced NRM in patients with a high risk of endothelial dysfunction ${ }^{45-47}$. UDCA has been shown to reduce early NRM and severe acute GVHD, but not late NRM nor cGVHD ${ }^{48}$. CXCL9 is an IFN response gene, and statins were shown to inhibit type I and type II IFNs and IFN responses ${ }^{49-52}$. Statins were reported to reduce cGVHD in mice ${ }^{53}$ and in CsAtreated patients ${ }^{54}$. However, evaluating the individual contributions of UDCA and statins to the protective effect is not possible in our cohort. In the current study, SEP was associated with reduced day +28 serum levels of CXCL9 and a lower incidence of severe cGVHD in high-risk patients. In addition, the risk of severe cGVHD was also lower in SEP-treated patients with high day +28 CXCL9. These results suggest that the favorable outcome of SEP on preventing severe cGVHD may be a result of the combined effects of immune modulation and endothelial protection. Nonetheless, the exact mechanism of how SEP may be able to protect patients from severe cGVHD is still elusive.

ATG is usually given from days -3 to -1 before alloSCT ${ }^{55}$. ATG significantly reduced the risk of severe cGVHD, but this effect was not different between highand low-risk groups. This suggests that ATG amended the effects of high day +28 CXCL9 on the incidence of cGVHD, rather than reducing the serum cytokine levels. All unrelated donors including mismatched donors received ATG in our study; therefore, we did not find any impact of HLA matching on severe cGVHD in multivariable models.

The main limitation of our study is represented by its retrospective and observational design making the findings vulnerable to unavoidable bias and unknown confounders. However, compared to published analyses on cGVHD-associated SNPs, our study includes an independent validation cohort, and suggests functional consequences of the SNPs on protein expression in the context of impeded suppressive effects by CNI. Knowing that the CXCL9 SNP status may improve patient management by promoting the use of endothelial protection strategies in high-risk patients.

In conclusion, the risk of severe cGVHD could be predicted by the genetic risk based on CXCR3 ligand genes in the absence of SEP. SEP may reduce the risk of severe CGVHD by regulating serum CXCL9 levels, and thus warrants further study.

\section{Acknowledgements}

We wish to acknowledge the great work of the physicians and the nursing staff of both transplant units, and, of course, the patients for making the study possible. We thank Michael Hess, Alexandra Hof, and Nic Geßner for their expert technical assistance and the construction and maintenance of the serum biobank. This work was supported by Deutsche

Forschungsgemeinschaft (TL820.8-1), Wilhelm-Sander-Stiftung (2016.077.1), B.L. U.T. e.V. (Weingarten), and by the EU's seventh framework program grant (agreement number 306240; SyStemAge). Doctoral student H.D. is sponsored by the China Scholarship Council.

\section{Author details \\ 'Department of Epidemiology, German Cancer Research Centre (DKFZ), Heidelberg, Germany. ${ }^{2}$ Division of Hematology, Oncology and \\ Tumorimmunology, Charité University Medicine Berlin, Berlin, Germany. ${ }^{3}$ Department of Medicine V, University Hospital Heidelberg, Heidelberg, Germany}

\section{Author contributions}

H.D. and S.P.R. designed and performed research, analyzed and discussed data, and wrote the paper. O.P., A.R., C.M.T., and P.D. treated patients, discussed data, and wrote the paper. R.K. designed and performed research, analyzed and discussed data, and wrote the paper. T.L. treated patients, collected clinical data, designed and performed research, analyzed and discussed data, and wrote the paper.

Funding

Open Access funding enabled and organized by Projekt DEAL.

\section{Conflict of interest}

The authors declare no competing interests.

\section{Publisher's note}

Springer Nature remains neutral with regard to jurisdictional claims in published maps and institutional affiliations.

Supplementary information The online version contains supplementary material available at https://doi.org/10.1038/s41408-021-00434-2.

Received: 4 September 2020 Revised: 17 December 2020 Accepted: 3 February 2021

Published online: 27 February 2021

\section{References}

1. Socie, G. et al. Long-term survival and late deaths after allogeneic bone marrow transplantation. Late Effects Working Committee of the International Bone Marrow Transplant Registry. N. Engl. J. Med. 341, 14-21 (1999).

2. Wingard, J. R. et al. Long-term survival and late deaths after allogeneic hematopoietic cell transplantation. J. Clin. Oncol. 29, 2230-2239 (2011).

3. Arai, S. et al. Increasing incidence of chronic graft-versus-host disease in allogeneic transplantation: a report from the Center for International Blood and Marrow Transplant Research. Biol. Blood Marrow Transplant. 21, 266-274 (2015).

4. Tyndall, A. \& Dazzi, F. Chronic GVHD as an autoimmune disease. Best Pract. Res. Clin. Haematol. 21, 281-289 (2008).

5. Dignan, F. L. et al. Diagnosis and management of chronic graft-versus-host disease. Br. J. Haematol. 158, 46-61 (2012).

6. Csanadi, M. et al. A systematic literature review of incidence, mortality, and relapse of patients diagnosed with chronic graft versus host disease. Expert Rev. Hematol. 12, 311-323 (2019).

7. Filipovich, A. H. et al. National Institutes of Health consensus development project on criteria for clinical trials in chronic graft-versus-host disease: I. Diagnosis and staging working group report. Biol. Blood Marrow Transplant. 11, 945-956 (2005).

8. Giesen, N. et al. CXCL9 predicts severity of chronic graft-versus-host disease. Transplantation. 104, 2354-2359 (2020). 
9. Chen, $\mathrm{X}$. et al. Absence of regulatory T-cell control of $\mathrm{TH} 1$ and $\mathrm{TH} 17$ cells is responsible for the autoimmune-mediated pathology in chronic graft-versushost disease. Blood 110, 3804-3813 (2007).

10. Bruggen, M. C. et al. Diverse T-cell responses characterize the different manifestations of cutaneous graft-versus-host disease. Blood 123, 290-299 (2014).

11. Nishimori, H. et al. Synthetic retinoid Am80 ameliorates chronic graft-versushost disease by down-regulating Th1 and Th17. Blood 119, 285-295 (2012).

12. Lacotte, S., Brun, S., Muller, S. \& Dumortier, H. CXCR3, inflammation, and autoimmune diseases. Ann. NY Acad. Sci. 1173, 310-317 (2009).

13. Sauty, A. et al. CXCR3 internalization following $T$ cell-endothelial cell contact preferential role of IFN-inducible T cell alpha chemoattractant (CXCL11). J. Immunol. 167, 7084-7093 (2001).

14. Loetscher, M., Loetscher, P., Brass, N., Meese, E. \& Moser, B. Lymphocyte-specific chemokine receptor CXCR3: regulation, chemokine binding and gene localization. Eur. J. Immunol. 28, 3696-3705 (1998).

15. Campanella, G. S. V. et al. Oligomerization of CXCL10 is necessary for endothelial cell presentation and in vivo activity. J. Immunol. 177, 6991-6998 (2006).

16. Lasagni, L. et al. An alternatively spliced variant of CXCR3 mediates the inhibition of endothelial cell growth induced by IP-10, Mig, and I-TAC, and acts as functional receptor for platelet factor 4. J. Exp. Med. 197, 1537-1549 (2003).

17. Vandercappellen, J., Van Damme, J. \& Struyf, S. The role of the CXC chemokines platelet factor-4 (CXCL4/PF-4) and its variant (CXCL4L1/PF-4var) in inflammation, angiogenesis and cancer. Cytokine Growth Factor Rev. 22, 1-18 (2011).

18. Groom, J. R. \& Luster, A. D. CXCR3 in T cell function. Exp. Cell Res. 317, 620-631 (2011).

19. Khoder, A. et al. Evidence for B cell exhaustion in chronic graft-versus-host disease. Front. Immunol. 8, 1937 (2017).

20. Green, L. A. et al. Cigarette smoke-induced CXCR3 receptor up-regulation mediates endothelial apoptosis. Am. J. Respir. Cell Mol. Biol. 47, 807-814 (2012).

21. Fleming, J. N. et al. Cutaneous chronic graft-versus-host disease does not have the abnormal endothelial phenotype or vascular rarefaction characteristic of systemic sclerosis. PLOS ONE 4, e6203 (2009).

22. Biedermann, B. C. et al. Endothelial injury mediated by cytotoxic T lymphocytes and loss of microvessels in chronic graft versus host disease. Lancet $\mathbf{3 5 9}$ 2078-2083 (2002)

23. Westekemper, H. et al. Differential chemokine expression in chronic GVHD of the conjunctiva. Bone Marrow Transplant. 45, 1340-1346 (2010).

24. Sugerman, P. B. et al. Kinetics of gene expression in murine cutaneous graftversus-host disease. Am. J. Pathol. 164, 2189-2202 (2004).

25. Croudace, J. E. et al. Chemokine-mediated tissue recruitment of CXCR3+ CD4+ T cells plays a major role in the pathogenesis of chronic GVHD. Blood 120, 4246-4255 (2012).

26. Rozmus, J. et al. Higher levels of free plasma mitochondrial DNA are associated with the onset of chronic GvHD. Bone Marrow Transplant. https:/doi.org/ 10.1038/s41409-018-0156-y (2018).

27. Imanguli, M. M. et al. Increased T-bet+ cytotoxic effectors and type I interferon-mediated processes in chronic graft-versus-host disease of the oral mucosa. Blood 113, 3620-3630 (2009).

28. Marini, B. L., Choi, S. W., Byersdorfer, C. A., Cronin, S. \& Frame, D. G. Treatment of dyslipidemia in allogeneic hematopoietic stem cell transplant patients. Biol. Blood Marrow Transplant. 21, 809-820 (2015).

29. Lentine, K. L. \& Brennan, D. C. Statin use after renal transplantation: a systematic quality review of trial-based evidence. Nephrol. Dial. Transplant. 19, 2378-2386 (2004).

30. Giesen, N. et al. CXCL9 predicts severity at onset of chronic graft-versus-host disease. Transplantation https://doi.org/10.1097/TP.0000000000003108 (2020).

31. Yu, J. et al. Biomarker panel for chronic graft-versus-host disease. J. Clin. Oncol. 34, 2583-2590 (2016).

32. Pratt, L. M. et al. IL15 levels on day 7 after hematopoietic cell transplantation predict chronic GVHD. Bone Marrow Transplant. 48, 722-728 (2013).

33. Cooke, K. R. et al. The biology of chronic graft-versus-host disease: a task force Report from the National Institutes of Health Consensus Development Project on Criteria for Clinical Trials in Chronic Graft-versus-Host Disease. Biol. Blood Marrow Transplant. 23, 211-234 (2017).

34. Alevizopoulos, A., Dusserre, Y., Ruegg, U. \& Mermod, N. Regulation of the transforming growth factor beta-responsive transcription factor CTF-1 by calcineurin and calcium/calmodulin-dependent protein kinase IV. J. Biol. Chem. 272, 23597-23605 (1997).

35. Blaeser, F., Ho, N., Prywes, R. \& Chatila, T. A. Ca(2+)-dependent gene expression mediated by MEF2 transcription factors. J. Biol. Chem. 275, 197-209 (2000),

36. Mao, Z. \& Wiedmann, M. Calcineurin enhances MEF2 DNA binding activity in calcium-dependent survival of cerebellar granule neurons. J. Biol. Chem. 274 31102-31107 (1999).

37. Wang, J. et al. Sequence features and chromatin structure around the genomic regions bound by 119 human transcription factors. Genome Res. 22, 1798-1812 (2012)

38. Fae, K. C et al. CXCL9/Mig mediates T cells recruitment to valvular tissue lesions of chronic rheumatic heart disease patients. Inflammation 36, 800-811 (2013).

39. Amatschek, S. et al. CXCL9 induces chemotaxis, chemorepulsion and endothelial barrier disruption through CXCR3-mediated activation of melanoma cells. Br. J. Cancer 104, 469-479 (2011).

40. McManigle, W., Youssef, A. \& Sarantopoulos, S. B cells in chronic graft-versushost disease. Hum. Immunol. 80, 393-399 (2019).

41. Sarantopoulos, S., Blazar, B. R., Cutler, C. \& Ritz, J. B cells in chronic graft-versushost disease. Biol. Blood Marrow Transplant. 21, 16-23 (2015).

42. Akiyama, Y. et al. Increased CXCR3 expression of infiltrating plasma cells in hunner type interstitial cystitis. Sci. Rep. 6, 28652 (2016).

43. Henneken, M., Dorner, T., Burmester, G. R. \& Berek, C. Differential expression of chemokine receptors on peripheral blood B cells from patients with rheumatoid arthritis and systemic lupus erythematosus. Arthritis Res. Ther. 7, R1001-R1013 (2005).

44. Marques, C. P. et al. CXCR3-dependent plasma blast migration to the central nervous system during viral encephalomyelitis. J. Virol. 85, 6136-6147 (2011).

45. Rachakonda, S. P. et al. Single nucleotide polymorphisms in CD40L predict endothelial complications and mortality after allogeneic stem-cell transplantation. J. Clin. Oncol. 36, 789-800 (2018).

46. Zeisbrich, M. et al. Transplant-associated thrombotic microangiopathy is an endothelial complication associated with refractoriness of acute GvHD. Bone Marrow Transplant. 52, 1399-1405 (2017).

47. Rachakonda, S. P. et al. Single-nucleotide polymorphisms within the thrombomodulin gene (THBD) predict mortality in patients with graft-versus-host disease. J. Clin. Oncol. 32, 3421-3427 (2014).

48. Ruutu, T. et al. Improved survival with ursodeoxycholic acid prophylaxis in allogeneic stem cell transplantation: long-term follow-up of a randomized study. Biol. Blood Marrow Transplant. 20, 135-138 (2014).

49. Amuro, H. et al. Statins, inhibitors of 3-hydroxy-3-methylglutaryl-coenzyme A reductase, function as inhibitors of cellular and molecular components involved in type I interferon production. Arthritis Rheum. 62, 2073-2085 (2010).

50. Jameel, A. et al. Statin modulation of human T-cell proliferation, IL-1beta and IL-17 production, and IFN-gamma T cell expression: synergy with conventional immunosuppressive agents. Int. J. Inflamm. 2013, 434586 (2013).

51. Lee, S. J., Qin, H. \& Benveniste, E. N. The IFN-gamma-induced transcriptional program of the CIITA gene is inhibited by statins. Eur. J. Immunol. 38 2325-2336 (2008)

52. Mach, F. Statins as immunomodulatory agents. Circulation 109, ॥15-|I17 (2004).

53. Yoon, H. K., Lim, J. Y., Kim, T. J., Cho, C. S. \& Min, C. K. Effects of pravastatin on murine chronic graft-versus-host disease. Transplantation 90, 853-860 (2010).

54. Rotta, M. et al. Impact of recipient statin treatment on graft-versus-host disease after allogeneic hematopoietic cell transplantation. Biol. Blood Marrow Transplant. 16, 1463-1466 (2010).

55. Theurich, S. et al. Anti-thymocyte globulins for post-transplant graft-versushost disease prophylaxis-A systematic review and meta-analysis. Crit. Rev. Oncol. Hematol. 88, 178-186 (2013). 